\title{
INCREASED SYSTEMIC IL-6 LEVELS POINT TO INFLAMMATION AS A DETERMINANT OF RENAL CELL CARCINOMA DEVELOPMENT
}

\author{
Giovana Cechim ${ }^{1}$, Joel Henrique Ellwanger ${ }^{1}$, Valéria de Lima \\ Kaminski ${ }^{1}$, Milton Berger ${ }^{2}$, José Artur Bogo Chies ${ }^{1}$
}

\begin{abstract}
Introduction: Renal cell carcinoma (RCC) is one of the most prevalent kidney tumors. Inflammation is believed to be a key factor in its progression and spread since inflammatory markers are generally associated with poor prognosis in RCC patients. Cytokines are cell communication molecules involved in both healthy and pathological processes, including tumor growth and progression. Recent findings suggest that cytokine level measurements could be used for cancer monitoring and prognosis.
\end{abstract}

Methods: This study characterized and compared the levels of different cytokines associated with the classical Th1, Th2, and Th17 immune responses in plasma samples from RCC patients $(n=25)$ and healthy controls $(n=29)$. Cytokine levels (IL-2, IL-4, IL-6, IL-10, TNF- $\alpha$, IFN- $y$, and IL-17A) were evaluated by flow cytometry using a BD Cytometric Bead Array (CBA) kit.

Results: No statistical differences in systemic IL-2, IL-4, IL-10, IL-17A, TNF, and INF-y levels were observed between RCC patients and controls $(p>0.05)$. However, higher systemic IL-6 levels were observed in RCC patients $(p=0.0034)$.

Conclusions: This study highlights the importance of assessing the impact of IL-6 on RCC pathogenesis and its potential role as a biomarker of disease progression.

Keywords: CBA; Cytokines; IL-6; Inflammation; Renal cancer

\section{INTRODUCTION}

Inflammation is one of the hallmarks of cancer biology ${ }^{1}$. Inflammatory processes have been associated with cancer-related mechanisms such as the proliferation and survival of malignant cells, angiogenesis, and metastasis ${ }^{2}$. Moreover, there is evidence showing a cytokine-mediated link between inflammation and cancer ${ }^{3}$. In the context of renal cell carcinoma (RCC), the presence of inflammation is generally associated with poor prognosis ${ }^{4,5}$. In addition to resistance to chemotherapy and radiation, features of RCC include an absence of early warning symptoms and variable clinical manifestations ${ }^{6}$.

T-helper (Th) lymphocytes show heterogeneous cell populations, playing essential roles in the immune system. These cells promote cell-mediated immunity by inducing pro- or anti-inflammatory reactions mainly through the secretion of different sets of cytokines that determine the direction of the immune response ${ }^{7}$. Interleukins (ILs) are a subset of cytokines that allow for both cell-signaling in the immune system and the generation of a tightly controlled and specific immune response. Distinct immune cell subsets, such as Th1, Th2, and Th17, produce and secrete ILs that will locate and attach to specific targets via cell surface receptors, triggering a cascade of events within the target cell and ultimately altering its behavior. In this context, ILs play a variety of immunomodulatory roles and can induce the maturation, differentiation, migration, and adhesion of immune cells ${ }^{8}$.

Clin Biomed Res. 2021;41(3):1-211

1 Departamento de Genética, Universidade Federal do Rio Grande do Sul. Porto Alegre, RS, Brasil.

2 Departamento de Urologia, Hospital de Clínicas de Porto Alegre. Porto Alegre, RS, Brasil.

Corresponding author: José Artur Bogo Chies. jabchies@terra.com.br Instituto de Biociências, Universidade Federal do Rio Grande do Sul Av. Bento Gonçalves, 9500 91509-900, Porto Alegre, RS, Brasil. 
Cechim et al.

The importance of pro-inflammatory mediators in the development of renal cancer has been increasingly recognized in recent years. An adequate microenvironment for tumor growth and development encompasses the inflammation-mediated recruitment of leukocytes, expression of tumor-promoting chemokines and cytokines, and induction of an angiogenic switch for tumor blood supply ${ }^{2,9}$. Inflammatory molecules can also mediate cell communication to favor tumor development, illustrating the crucial role of cancerrelated inflammation. As they grow, solid malignant masses often lose their blood supply, resulting in inadequate oxygen and nutrient levels and leading to necrotic cell death and the release of pro-inflammatory mediators. This phenomenon establishes a cycle that induces neo-angiogenesis, allowing for the continued growth of remaining cancer cells ${ }^{9}$.

Interestingly, tumor progression requires strategies to avert immune system recognition through the establishment of an adequate tumor microenvironment ${ }^{10}$. Tumors can secrete factors that suppress and disrupt T-cell responsiveness, including immunosuppressive cytokines ${ }^{11}$. Another immune escape mechanism found in tumors involves the adaptive immune response ${ }^{8}$. ILs in the tumor microenvironment interact with various biomolecules and cell subpopulations, such as cancer stem cells, microRNA, epithelialmesenchymal transition markers, and transcription factors ${ }^{12}$. These interactions have been translated into several cytokine-based approaches for cancer monitoring and therapy.

Although cytokines are mainly involved in the tumor microenvironment, systemic inflammation levels can also provide important information on tumor and cancer progression ${ }^{2}$. According to the literature, plasma levels of inflammatory cytokines are associated with poor prognosis in patients with $\mathrm{RCC}^{2,12-15}$. The comprehension of the biological impact of individual cytokines on tumor progression is essential for the development of new biomarkers and possibly new therapeutic targets. Thus, considering the role of cytokines in cancer and the recent advances in RCC progression monitoring, the aim of this study was to evaluate the plasma levels of Th1, Th2, and Th17 cytokines in pre-treatment patients with RCC and healthy controls.

\section{METHODS}

\section{Patients and ethical concerns}

Pre-treatment patients diagnosed with RCC (Cancer group, $n=25$ ) were recruited at the Urology Service of the Hospital de Clínicas de Porto Alegre, located in the metropolitan region of the state of Rio Grande do Sul (southern Brazil). The diagnosis of renal cancer was confirmed by imaging and histopathological analysis. Healthy individuals with no cancer history (Control group, $n=29$ ) were also recruited in southern Brazil. These participants had a similar age to the individuals in the RCC group. This study was approved by the ethics committees of the Hospital de Clínicas de Porto Alegre and Universidade Federal do Rio Grande do Sul (CAAE No. 11858512.3.0000.5327). All participants signed a consent form in accordance with Resolution No. 466/2012 from the Ministério da Saúde ${ }^{16}$

\section{Tumor classification and plasma samples}

Tumors were classified by histopathological analysis according to two systems: I. Fuhrman System ${ }^{17}$; briefly lesions are classified into four grades (G1, G2, G3, and G4) according to nuclear size and presence of nucleoli ${ }^{18}$. II. TNM Classification of Malignant Tumors/TNM staging; originally proposed by Denoix ${ }^{19}$, this system is adopted by the Union for International Cancer Control (UICC) ${ }^{20}$. "T" refers to the primary tumor, "N" refers to regional lymph node involvement, and " $\mathrm{M}$ " refers to the presence or absence of distant metastasis ${ }^{20}$. This system classifies primary tumors $(T)$ into four stages according to their size and extent. Each stage can be divided into subcategories (a, b, c). For this study, we considered only the primary tumor ( $\mathrm{T}$ ) classification, since $\mathrm{N}$ and $M$ classifications were unavailable or not applicable to many of the tumors investigated.

Plasma samples were obtained from peripheral blood by centrifugation, after which the samples were kept under refrigeration $\left(-80^{\circ} \mathrm{C}\right)$ until the cytokine analyses. Of note, samples of individuals from the Cancer group were collected at the hospital on the day of tumor excision, prior to any other treatment.

\section{CBA analysis}

Cytokine levels were evaluated by flow cytometry using the BD Cytometric Bead Array (CBA) Human Th1/Th2/Th17 Cytokine kit (Catalog No. 560484, BD Biosciences, San Jose, CA, USA) at the Laboratory of Immunobiology and Immunogenetics (UFRGS, Brazil). This kit allows for the simultaneous detection of the following cytokines: IL-2, IL-4, IL-6, IL-10, TNF- $\alpha$, IFN- $\gamma$, and IL-17A. CBA analysis was performed using plasma samples, according to the manufacturer's instructions. The FCAP Array software (BD Biosciences) was used to analyze the raw data obtained with the FACSDiva software (BD Biosciences). Cytokine levels were expressed in $\mathrm{pg} / \mathrm{mL}$.

\section{Statistical analysis}

Once age was confirmed to be normally distributed, it was compared between groups using unpaired t-tests. Sex ratios were compared using Pearson's chi-square with Yates' correction. Cytokine levels did not show a normal distribution and were therefore 
compared through non-parametric tests. The KruskalWallis test was used for comparisons involving three groups and the Mann-Whitney $U$ test was used for comparisons between two groups. A p-value $<0.05$ was defined as statistically significant. The analyses were performed using WINPEPI ${ }^{21}$ and GraphPad Prism 5.01 (GraphPad Software, Inc., San Diego, CA, USA). The latter was also used for plotting graphs.

\section{RESULTS}

Table 1 shows the age and sex distribution of both groups. Neither variable differed significantly between the Cancer and Control groups $(p>0.05)$. Table 1 also shows the distribution of tumors according to TNM staging. The Fuhrman classification could not be made in three cases due to unavailable data.

Table 1: Characteristics of participants included in the study.

\begin{tabular}{|c|c|c|c|c|}
\hline \multicolumn{2}{|l|}{ Characteristic } & \multirow{2}{*}{$\begin{array}{c}\text { Cancer group } \\
(\mathbf{n}=\mathbf{2 5})\end{array}$} & \multirow{2}{*}{$\begin{array}{c}\text { Control group } \\
(\mathbf{n}=\mathbf{2 9})\end{array}$} & \multirow{2}{*}{$\frac{p \text {-value }}{}$} \\
\hline Age, years; median (IQR) & & & & \\
\hline \multirow{3}{*}{ Sex, n (\%) } & Male & $14(56.00)$ & $14(48.28)$ & \multirow{9}{*}{$0.769^{c}$} \\
\hline & Female & $11(44.00)$ & $15(51.72)$ & \\
\hline & T1a & $11(44.00)$ & - & \\
\hline \multirow[t]{3}{*}{ TNM staging, $\mathbf{n}(\%)$} & T1b & $5(20.00)$ & - & \\
\hline & T3a & $9(36.00)$ & - & \\
\hline & G1 & $1(4.55)$ & - & \\
\hline \multirow{3}{*}{ Fuhrman System, n (\%a) } & G2 & $13(59.09)$ & - & \\
\hline & G3 & $6(27.27)$ & - & \\
\hline & G4 & $2(9.09)$ & - & \\
\hline
\end{tabular}

IQR: interquartile range; $n$ : sample size; a: based on $n=22$; b: Unpaired t-test; c: Pearson's chi-square with Yates' correction.

Table 2 shows the levels of IL-2, IL-4, IL-6, IL-10, TNF- $\alpha$, IFN-y, and IL-17A in both groups. IL- 6 levels were significantly higher in the Cancer group compared to the Control group $(p=0.0034)$. No statistically significant differences were observed between the groups on any of the other cytokines $(p>0.05)$.

Table 2: Cytokine levels in cancer group and control group.

\begin{tabular}{lccc}
\hline \multicolumn{1}{c}{ Cytokine } & $\begin{array}{c}\text { Cancer group } \\
\mathbf{p g} / \mathbf{m L} \text {, median (IQR) } \\
(\mathbf{n}=\mathbf{2 5})\end{array}$ & $\begin{array}{c}\text { Control group } \\
\mathbf{p g} / \mathbf{m L} \text {, median (IQR) } \\
(\mathbf{n}=\mathbf{2 9})\end{array}$ & $\begin{array}{c}\boldsymbol{p} \text {-value } \\
\text { (Mann-Whitney U test) }\end{array}$ \\
\hline IL-2 & $0.0(0.0-0.14)$ & $0.0(0.0-0.0)$ & 0.7300 \\
IL-4 & $0.0(0.0-0.0)$ & $0.0(0.0-0.075)$ & 0.2389 \\
IL-6 & $2.30(1.06-4.47)$ & $0.85(0.65-1.72)$ & $\mathbf{0 . 0 0 3 4}$ \\
IL-10 & $0.78(0.17-1.60)$ & $0.58(0.305-0.765)$ & 0.2414 \\
IL-17A & $0.0(0.0-0.0)$ & $0.0(0.0-0.0)$ & 0.4543 \\
TNF & $0.0(0.0-0.83)$ & $0.0(0.0-0.68)$ & 0.6286 \\
IFN-y & $0.0(0.0-0.135)$ & $0.0(0.0-0.02)$ & 0.2868 \\
\hline
\end{tabular}

IQR: interquartile range. Significant $p$-value is shown in bold.

Since only IL-6 and IL-10 showed median values $>0 \mathrm{pg} / \mathrm{mL}$, these two cytokines were chosen for subsequent analyses involving the stratification of individuals in the Cancer group according to TNM staging and Fuhrman grades. Patients with Fuhrman grades $\mathrm{G} 1$ and $\mathrm{G} 2$ were included in one group while those with grades $\mathrm{G} 3$ and $\mathrm{G} 4$ were placed in a second group. This procedure was adopted due to the small number of individuals classified as $G 1(n=1)$ and G4 $(n=2)$. However, no statistically significant differences $(p>0.05)$ were found in IL-6 and IL-10 levels between tumor grade groups (Figure 1). 

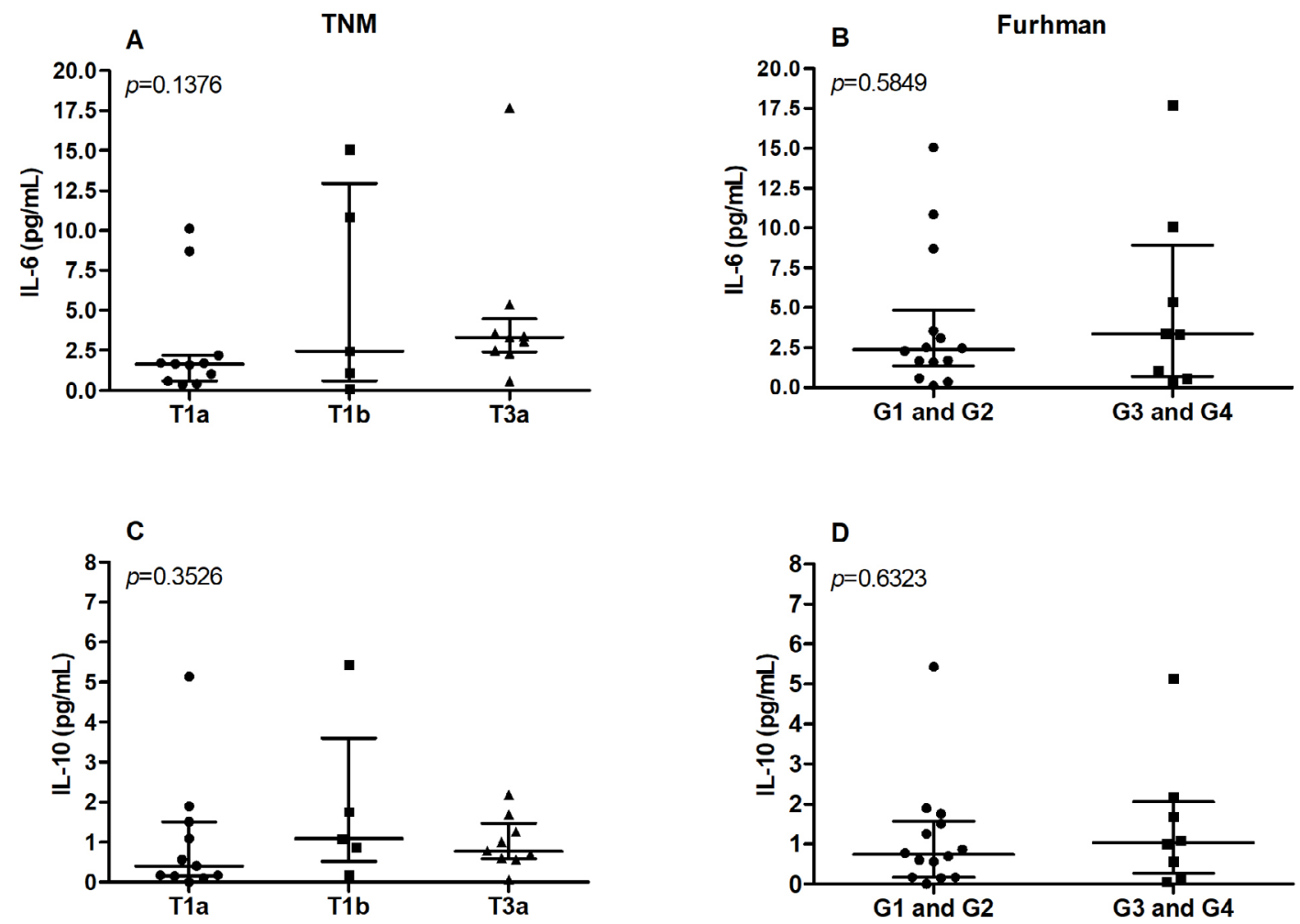

Figure 1: Comparison of IL-6 and IL-10 levels in cancer patients according to TNM $(n=25)$ and Furhman scale $(n=22)$. Kruskal-Wallis test was used for comparisons between TNM stages. Mann-Whitney $U$ test was used for comparisons between Furhman grades.

\section{DISCUSSION}

In this study, we measured plasma levels of IL-2, IL-4, IL-6, IL-10, IL-17A, TNF, and IFN- $\alpha$ in patients with renal cancer. Our results indicated that IL-6 levels were increased in RCC patients in the pre-treatment stage. However, these levels did not differ between patients with tumors of different histopathological grades.

Inflammation plays an important role in the initiation of malignant transformation and cancer progression ${ }^{2,9,22}$. Furthermore, the presence of inflammatory cytokines such as TNF- $\alpha$ and IL- 6 can increase the invasive capacity of malignant cells ${ }^{23-26}$. In this study, only IL-6 was present at significantly higher levels in renal cancer patients compared to controls. This result adds to the growing evidence of the role of IL- 6 in the development and progression of renal cancer ${ }^{6,27}$. IL-6 promotes cell proliferation, migration, and invasion mainly by the activation of transcription factors STAT3 and NF-KB ${ }^{28-31}$. IL-6 has also been found to modulate angiogenesis in RCC by increasing the expression of HIF1 $\alpha$ and VEGF ${ }^{32}$.

IL-6 is a pro-inflammatory cytokine with multiple immune regulatory functions. It is produced by a variety of cells, including macrophages, $T$ and B cells, fibroblasts, endothelial cells, and epithelial cells, particularly renal mesangial and tubular cells $\mathbf{s}^{12,33}$. IL-6 function is largely mediated by two membrane proteins: an $80 \mathrm{kDa}$ binding receptor (IL-6R) and the signal transducer gp130 protein. The soluble IL-6 receptor gp80 (sIL-6R) binds to circulating IL-6 molecules forming the IL-6/sIL-6R complex, which can then bind to and activate the gpl30 transducer chain in any cell expressing the gp130 receptor subunit ${ }^{34}$.

Considering the link between inflammation and cancer, it is important to highlight that renal carcinoma cells exposed to hypoxia can secrete IL-6, and increased local IL-6 expression promotes carcinoma cell invasion ${ }^{15}$. Indeed, elevated IL-6 mRNA expression levels and IL- 6 secretion have been detected in primary RCC tissues ${ }^{6}$. In this same direction, IL-6 mRNA and IL-6 protein receptors have been identified in both primary RCC cultures and established RCC cell lines ${ }^{6,35}$. In a study by Sakai et al. ${ }^{36}$, high serum levels of IL- 6 were observed in patients with RCC both before and after nephrectomy. The presence of abnormally high IL-6 serum levels in patients with metastatic RCC has been suggested as a potential 
independent prognostic factor for these individuals ${ }^{14}$. Blay et al. ${ }^{37}$ investigated the potential role of circulating IL-6 in the paraneoplastic inflammatory and cholestatic syndrome associated with metastatic RCC. In their study, a correlation between the presence of serum IL-6 and systemic symptoms was observed, as were increased serum levels of C-reactive protein and haptoglobin ${ }^{37}$. Costes et al ${ }^{38}$ showed a correlation between the size and stage of RCC with serum IL-6 concentration. Also, IL-6 levels correlated with a worse prognosis in patients with metastatic $\mathrm{RCC}^{39}$.

Of note, in our study, the patient with the highest IL-6 levels was also the only one with metastasis, presenting with lymph node involvement. This corroborates the previously mentioned association between IL- 6 and a higher invasive capacity of malignant cells. Thus, the measurement of IL-6 plasma levels in patients with metastatic renal cancer might represent a complementary approach to the evaluation or prediction of the response to immunotherapy. Importantly, the most common drugs used in the treatment of metastatic renal cancer (for example, Sunitinib, Bevacizumab, Pazopanib and Sorafenib) inhibit tyrosine kinase and STAT3 signaling pathways, both of which can be directly activated by IL-6 $6^{40,41}$.

The IL-6/sIL-6R complex has been implicated in various deleterious effects of IL- 6 in chronic inflammatory diseases and cancer ${ }^{34}$. This could explain the elevated levels of this IL in RCC and other cancer types, with and without metastasis, observed by our group and other researchers. In the tumor microenvironment, tumor endothelial cells up-regulate the expression of gp130, down-regulate the expression of membrane-bound IL-6R, and are targeted by the IL-6/IL-6sR complex, which leads to cell proliferation, inhibition of apoptosis, and enhanced carcinogenesis ${ }^{42}$. Furthermore, as gp130 is expressed in almost every human cell, the IL-6/sIL-6R complex can affect many circulating cells, which can further explain the high levels of this IL in RCC, other types of cancer, and chronic inflammatory diseases ${ }^{6,43}$.

In conclusion, considering the important role of IL- 6 in the establishment and maintenance of renal cancer, immunotherapeutic drugs that directly interfere with IL-6 signaling pathways may constitute a promising alternative for the treatment of metastatic renal cancer. Some of these drugs [Tocilizumab (antihuman IL-6R) and Siltuximab (anti-IL-6 monoclonal antibody)] are already used to treat other diseases and could potentially be tested for the treatment of renal cancer ${ }^{6,30,44-46}$. In summary, the higher systemic IL-6 levels observed in RCC patients point to the importance of future studies of this IL, with a special focus on IL-6-targeted therapeutic strategies and the role of IL-6 in the establishment, development, and progression of cancer.

\section{Acknowledgments and funding}

This study received financial support from the Hospital de Clínicas de Porto Alegre (HCPA, Porto Alegre, Brazil), Conselho Nacional de Desenvolvimento Científico e Tecnológico (CNPq, Brazil; Grant No. 473115/2011-5 and 305839/2015-2), and Coordenação de Aperfeiçoamento de Pessoal de Nível Superior (CAPES, Brazil). GC, JHE, and VLK received doctoral fellowships from Capes (Brazil). $\mathrm{JABC}$ has a research fellowship from CNPq (Brazil). Currently, JHE has a postdoctoral fellowship from CAPES (Programa Nacional de Pós-Doutorado-PNPD) and VLK has a postdoctoral fellowship from the Fundação de Amparo à Pesquisa do Estado de São Paulo (Fapesp).

\section{Author contributions}

GC and MB collected the samples and data from participants in the study. GC, JHE, and VLK performed the analyses and wrote the first version of the manuscript. JABC supervised the work and revised the manuscript.

\section{Conflicts of interest}

The authors declare no conflicts of interest regarding this study.

\section{REFERENCES}

1. Hanahan D, Weinberg RA. Hallmarks of cancer: the next generation. Cell. 2011;144(5):646-74.

2. Mantovani A, Allavena P, Sica A, Balkwill F. Cancer-related inflammation. Nature. 2008;454:436-44.

3. Lin WW, Karin M. A cytokine-mediated link between innate immunity, inflammation, and cancer. J Clin Invest. 2007;117(5):1175-83.
4. Yoshida N, Ikemoto S, Narita K, Sugimura K, Wada S, Yasumoto R, et al. Interleukin-6, tumour necrosis factor alpha and interleukin-1beta in patients with renal cell carcinoma. Br J Cancer 2002;86(9):1396-400.

5. Cuadros T, Trilla E, Sarro E, Vila MR, Vilardell J, Torres I, et al. HAVCR/KIM1 activates the IL-6/STAT-3 pathway in clear cell renal cell carcinoma and determines tumor progression and patient outcome. Cancer Res. 2014;74(5):1416-28.

6. Kaminska K, Czarnecka AM, Escudier B, Lian F, Szczylik C. Interleukin-6 as an emerging regulator of renal cell cancer. Urol Oncol. 2015;33(11):476-85.

7. Annunziato F, Romagnani S. Heterogeneity of human effector CD4+ T cells. Arthritis Res Ther. 2009;11(6):257. 
8. Anestakis $D$, Petanidis $S$, Kalyvas $S$, Nday CM, Tsave O, Kioseoglou E, et al. Mechanisms and applications of interleukins in cancer immunotherapy. Int J Mol Sci. 2015;16(1):1691-710.

9. Grivennikov SI, Greten FR, Karin M. Immunity, inflammation, and cancer. Cell. 2010;140(6):883-99.

10. Bhatia A, Kumar Y. Cellular and molecular mechanisms in cancer immune escape: a comprehensive review. Expert Rev Clin Immunol. 2014;10(1):41-62.

11. Mao Y, Poschke I, Kiessling R. Tumour-induced immune suppression: role of inflammatory mediators released by myelomonocytic cells. J Intern Med. 2014;276(2):154-70.

12. Setrerrahmane $\mathrm{S}, \mathrm{XuH}$. Tumor-related interleukins: old validated targets for new anti-cancer drug development. Mol Cancer. 2017;16(1):153.

13. Dosquet C, Schaetz A, Faucher C, Lepage E, Wautier JL, Richard F, et al. Tumour necrosis factor- $\alpha$, interleukin$1 \beta$ and interleukin- 6 in patients with renal cell carcinoma. Eur $J$ Cancer. 1994;30A(2):162-7.

14. Negrier S, Perol D, Menetrier-Caux $C$, Escudier B, Pallardy M, Ravaud A, et al. Interleukin-6, interleukin-10, and vascular endothelial growth factor in metastatic renal cell carcinoma: prognostic value of interleukin-6--from the Groupe Français d'Immunotherapie. $J$ Clin Oncol. 2004;22(12):2371-8.

15. Fitzgerald JP, Nayak $B$, Shanmugasundaram K, Friedrichs $\mathrm{W}$, Sudarshan S, Eid AA, et al. Nox4 mediates renal cell carcinoma cell invasion through hypoxia-induced interleukin 6- and 8- production. PLOS One. 2012;7(1):e30712.

16. Brasil. Ministério da Saúde. Conselho Nacional de Saúde. Resolução $n^{\circ}$ 466, de 12 de dezembro de 2012. Brasília, DF; 2012.

17. Fuhrman SA, Lasky LC, Limas L. Prognostic significance of morphologic parameters in renal cell carcinoma. Am J Surg Pathol. 1982;6(7):655-63.

18. Ficarra V, Martignoni G, Maffei N, Brunelli M, Novara G, Zanolla L, et al. Original and reviewed nuclear grading according to the Fuhrman system: a multivariate analysis of 388 patients with conventional renal cell carcinoma. Cancer. 2005;103(1):68-75.
19. Denoix PF. Enquete permanent dans les centres anticancéreaux. Bull Inst Nat Hyg. 1946;1:70-5.

20. Union for International Cancer Control. TNM [Internet]. c2021 [cited 2021 May 21] Available from: https://www.uicc. org/resources/tnm

21. Abramson JH. WINPEPI updated: computer programs for epidemiologists, and their teaching potential. Epidemiol Perspect Innov. 2011;8(1):1.

22. Trinchieri G. Cancer and inflammation: an old intuition with rapidly evolving new concepts. Annu Rev Immunol. 2012;30:677-706.

23. Xie TX, Wei D, Liu M, Gao AC, Ali-Osman F, Sawaya R, et al. Stat3 activation regulates the expression of matrix metalloproteinase-2 and tumor invasion and metastasis. Oncogene. 2004;23(2):3550-60.

24. Sullivan NJ, Sasser AK, Axel AE, Vesuna F, Raman V, Ramirez N, et al. Interleukin-6 induces an epithelialmesenchymal transition phenotype in human breast cancer cells. Oncogene. 2009;28(33):2940-7.

25. Elinav E, Nowarski R, Thaiss CA, $\mathrm{Hu} \mathrm{B}$, Jin C, Flavell RA. Inflammationinduced cancer: crosstalk between tumours, immune cells and microorganisms. Nat Rev Cancer. 2013;13:759-71.

26. Cathcart JM, Banach A, Liu A, Chen J, Goligorsky M, Cao J. Interleukin-6 increases matrix metalloproteinase-14 (MMP-14) levels via down-regulation of p53 to drive cancer progression. Oncotarget. 2016;7(38):61107-20.

27. Gudbrandsdottir $\mathrm{G}$, Aarstad $\mathrm{HH}$, Bostad L, Hjelle KM, Aarstad HJ, Bruserud $O$, et al. Serum levels of the IL-6 family of cytokines predict prognosis in renal cell carcinoma (RCC). Cancer Immunol Immunother. 2021;70(1):19-30.

28. Lee H, Herrmann A, Deng JH, Kujawski M, Niu G, Li Z, et al. Persistently activated Stat3 maintains constitutive NF-kappaB activity in tumors. Cancer Cell. 2009;15(4):283-93.

29. Yu H, Pardoll D, Jove R. STATs in cancer inflammation and immunity: a leading role for STAT3. Nat Rev Cancer. 2009;9(11):798-809.
30. Johnson DE, O'Keefe RA, Grandis JR. Targeting the IL-6/JAK/STAT3 signalling axis in cancer. Nat Rev Clin Oncol. 2018;15(4):234-48.

31. Taniguchi K, Karin M. NF-KB, inflammation, immunity and cancer: coming of age. Nat Rev Immunol. 2018;18(5):309-24.

32. Santoni M, Conti A, Piva F, Massari F, Ciccarese C, Burattini L, et al. Role of STAT3 pathway in genitourinary tumors. Future Sci OA. 2015;1(3):FSO15.

33. Kishimoto T. The biology of interleukin-6. Blood. 1989;74(1):1-10.

34. Nguyen DP, Li J, Tewari AK. Inflammation and prostate cancer: the role of interleukin 6 (IL-6). BJU Int. 2014;113(6):986-92.

35. Takenawa J, Kaneko Y, Fukumoto M, Fukatsu A, Hirano T, Fukuyama $\mathrm{H}$, et al. Enhanced expression of interleukin-6 in primary human renal cell carcinomas. J Natl Cancer Inst. 1991;83(22):1668-72.

36. Sakai A, Kawano M, Kuramoto A. Interleukin-6 produced by renal-cell carcinoma cells and progression of multiple myeloma. N Engl J Med. 1991;324(26):1893-4.

37. Blay JY, Rossi JF, Wijdenes J, Menetrier-Caux C, Schemann S, Negrier S, et al. Role of interleukin-6 in the paraneoplastic inflammatory syndrome associated with renalcell carcinoma. Int J Cancer. 1997;72(3):424-30.

38. Costes V, Liautard J, Picot MC, Robert M, Lequeux N, Brochier J, et al. Expression of the interleukin 6 receptor in primary renal cell carcinoma. J Clin Pathol. 1997;50(1):835-40.

39. Stadler WM, Richards JM, Vogelzang NJ. Serum interleukin-6 levels in metastatic renal cell cancer: correlation with survival but not an independent prognostic indicator. J Natl Cancer Inst. 1992;84(23):1835-6.

40. Hodge DR, Hurt EM, Farrar WL. The role of IL- 6 and STAT3 in inflammation and cancer. Eur J Cancer. 2005;41(16):2502-12.

41. Ranieri G, Marech I, Asabella AN, Di Palo A, Porcelli M, Lavelli V, et al. Tyrosine-kinase inhibitors therapies with mainly anti-angiogenic activity in 
advanced renal cell carcinoma: value of PET/CT in response evaluation. Int J Mol Sci. 2017;18(9):1937.

42. Matsumoto S, Hara T, Mitsuyama K, Yamamoto M, Tsuruta O, Sata M, et al. Essential roles of IL-6 trans-signaling in colonic epithelial cells, induced by the IL-6/soluble-IL-6 receptor derived from lamina propria macrophages, on the development of colitis-associated premalignant cancer in a murine model. J Immunol. 2010;184(3):1543-51.
43. Uciechowski P, Dempke WCM. Interleukin-6: a masterplayer in the cytokine network. Oncology. 2020;98(3):131-7.

44. Oguro $T$, Ishibashi $K$, Sugino $T$, Hashimoto K, Tomita S, Takahashi N, et al. Humanised antihuman IL-6R antibody with interferon inhibits renal cell carcinoma cell growth in vitro and in vivo through suppressed SOCS3 expression. Eur J Cancer. 2013;49(7):1715-24.
45. Ishibashi K, Haber T, Breuksch I, Gebhard S, Sugino T, Kubo H, et al. Overriding TKI resistance of renal cell carcinoma by combination therapy with IL-6 receptor blockade. Oncotarget. 2017;8(33): 55230-45.

46. Garbers C, Heink S, Korn T, RoseJohn S. Interleukin-6: designing specific therapeutics for a complex cytokine. Nat Rev Drug Discov. 2018;17(6):395-412. 